\title{
Uncertain random time-cost trade-off problem
}

\author{
Hua Ke
}

\section{Correspondence:}

hke@tongji.edu.cn

School of Economics and

Management, Tongji University,

Shanghai 200092, China

\begin{abstract}
Since for most projects, 'new' parts (with little or no historical data) and 'old' parts (with sufficient historical data) always coexist in the same project, uncertainty and randomness should be considered simultaneously in time-cost trade-off problem. In this paper, combined with uncertainty theory and dependent-chance programming, an uncertain random time-cost trade-off model is built. A crisp equivalent model is also given for some special case. Besides, uncertain random simulation and genetic algorithm are integrated for solving the proposed model.
\end{abstract}

Keywords: Project scheduling; Time-cost trade-off; Uncertain variable; Uncertain random programming; Dependent-chance programming

\section{Introduction}

Time-cost trade-off problem (TCTP), a specific type of project scheduling problem, desires to balance two important goals in real-life projects, project completion times, and project total costs to achieve decision-making requirements. For real-life projects, project managers always consider the trade-offs among the performance goals (e.g., project cost, project completion time, project quality, etc.) for project scheduling and control. The time-cost trade-off problem takes into account the project time-cost trade-off by crashing or prolonging project activity durations. In 1961, Kelly [1] first did research on the TCTP. In the following years, many researches have been done on the TCTP with assumption of deterministic activity durations [2-5].

In real-life projects, activity durations may be variational for many external factors, such as technological advance, weather change, labor force shortage, etc. For some projects, since many similar projects have ever been fulfilled before, there are enough historical data for deducing probability distributions for describing imprecise quantities in projects. Hence, probability theory was introduced into the TCTP for estimating nondeterministic factors. In 1985, Wollmer [6] discussed a stochastic version of the deterministic linear TCTP. Following that, many authors discussed the TCTP with stochastic environmental factors [7-9].

In project management, one of the most important characteristics of the concept 'project' is that there is always something new in a project. That is, there are always some parts which are never performed before or only similarly performed. For such parts in projects, historical data for estimating probability distributions of some imprecise quantities are always not enough when facing external indeterminacy, which means that probability theory is no longer valid for describing imprecise quantities (e.g., activity

(c) 2014 Ke; licensee Springer. This is an Open Access article distributed under the terms of the Creative Commons Attribution License (http://creativecommons.org/licenses/by/4.0), which permits unrestricted use, distribution, and reproduction in any medium, provided the original work is properly credited. 
durations) in such project parts. For this case, project activity durations were originally described by fuzzy variables, and many researchers studied the TCTP with fuzzy parameters [10-13].

In the past years, subjective probability theory and fuzzy set theory were applied to estimate imprecise quantities (or indeterminacy) with no or little statistical data in decision systems, including project scheduling and control. However, actually both these two theories were proved not suitable for the above case, illustrated by some examples [14]. Since such a type of indeterminacy cannot be depicted via randomness or fuzziness, a new theory is needed. On this occasion, Liu founded uncertainty theory in 2007 [15] and refined it in 2010 [16], which is a branch of axiomatic mathematics for modeling human uncertainty. Since its foundation, uncertainty theory has been widely applied in many decision systems, such as portfolio selection [17], inventory problem [18], facility location [19], supply chain integrated production-inventory problem [20], investment problem [21], differential games [22], and coalitional game [23]. Especially, Ke [24] applied uncertain measure for estimating activity durations in time-cost trade-off problem.

For most projects, though they always include some 'new' parts, meanwhile they may contain some common parts with many other finished projects. With enough statistical data from other projects, probability theory may be introduced to estimate imprecise quantities in these common parts. Then for many projects, integrated by some 'new' parts and some other 'old' (common) parts, randomness and uncertainty may coexist. For such cases, the concepts of uncertain random variable and chance measure and the philosophy of uncertain random programming were presented by Liu [25] and Liu [26], respectively. Uncertain random programming has been further studied in some aspects, e.g., uncertain random graph and uncertain random network [27], uncertain random risk analysis [28], uncertain random multi-objective optimization [29], and uncertain random multilevel programming [30]. In this paper, we introduce chance measure and uncertain random programming to model the TCTP with coexisted randomness and uncertainty. As Huang and Ding [31] illustrated that the standard path algorithms (e.g., the well-known Dijkstra method) were not able to arrive at solutions for searching critical path of this problem, genetic algorithm (GA) is applied in this paper.

The remainder of this paper is organized as follows: in Section 'Preliminaries of uncertainty theory', some concepts and useful theorems of uncertainty theory are presented. Section 'Description of time-cost trade-off problem' briefly describes the problem with uncertain random parameters. In Section 'Uncertain random time-cost trade-off model with dependent-chance programming', with dependent-chance programming, an uncertain random time-cost trade-off model is built and a crisp TCTP model is also given for some special case of uncertain random variable. Section 'Algorithm description with numerical experiment' conducts a numerical experiment to illustrate the proposed model. Finally, Section 'Conclusions' draws some concluding statements.

\section{Preliminaries of uncertainty theory}

In this section, we introduce some basic concepts and theorems for building uncertain random TCTP model. Let $\Gamma$ be a nonempty set, $\mathcal{L}$ a $\sigma$-algebra over $\Gamma$, and each element $\Lambda$ in $\mathcal{L}$ is called an event. 
Definition 1. (Liu [15]) The set function $\mathcal{M}$ is called an uncertain measure if it satisfies:

Axiom 1 (Normality Axiom). $\mathcal{M}\{\Gamma\}=1$ for the universal set $\Gamma$.

Axiom 2 (Duality Axiom). $\mathcal{M}\{\Lambda\}+\mathcal{M}\left\{\Lambda^{c}\right\}=1$ for any event $\Lambda$.

Axiom 3 (Subadditivity Axiom). For every countable sequence of events $\Lambda_{1}, \Lambda_{2}, \cdots$, we have

$$
\mathcal{M}\left\{\bigcup_{i=1}^{\infty} \Lambda_{i}\right\} \leq \sum_{i=1}^{\infty} \mathcal{M}\left\{\Lambda_{i}\right\}
$$

Besides, the product uncertain measure on the product $\sigma$-algebra $\mathcal{L}$ was defined by Liu [32] as follows:

Axiom 4 (Product Axiom). Let $\left(\Gamma_{k}, \mathcal{L}_{k}, \mathcal{M}_{k}\right)$ be uncertainty spaces for $k=1,2, \cdots$. The product uncertain measure $\mathcal{M}$ is an uncertain measure satisfying

$$
\mathcal{M}\left\{\prod_{k=1}^{\infty} \Lambda_{k}\right\}=\bigwedge_{k=1}^{\infty} \mathcal{M}_{k}\left\{\Lambda_{k}\right\}
$$

where $\Lambda_{k}$ are arbitrarily chosen events from $\mathcal{L}_{k}$ for $k=1,2, \cdots$, respectively.

Definition 2. (Liu [15]) An uncertain variable is a measurable function $\xi$ from an uncertainty space $(\Gamma, \mathcal{L}, \mathcal{M})$ to the set of real numbers, i.e., for any Borel set $B$ of real numbers, the set

$$
\{\xi \in B\}=\{\gamma \in \Gamma \mid \xi(\gamma) \in B\}
$$

is an event.

Definition 3. (Liu [32]) The uncertain variables $\xi_{1}, \xi_{2}, \cdots, \xi_{n}$ are said to be independent if

$$
\mathcal{M}\left\{\bigcap_{i=1}^{n}\left(\xi_{i} \in B_{i}\right)\right\}=\bigwedge_{i=1}^{n} \mathcal{M}\left\{\xi_{i} \in B_{i}\right\}
$$

for any Borel sets $B_{1}, B_{2}, \cdots, B_{n}$.

Sometimes, to describe real-world optimization problems with uncertain parameters, it is sufficient to know the uncertainty distribution rather than the uncertain variable itself.

Definition 4. (Liu [15]) The uncertainty distribution $\Phi$ of an uncertain variable $\xi$ is defined by

$$
\Phi(x)=\mathcal{M}\{\xi \leq x\}
$$

for any real number $x$.

Definition 5. (Liu [16]) An uncertainty distribution $\Phi(x)$ is said to be regular if it is a continuous and strictly increasing function with respect to $x$ at which $0<\Phi(x)<1$, and

$$
\lim _{x \rightarrow-\infty} \Phi(x)=0, \quad \lim _{x \rightarrow+\infty} \Phi(x)=1 .
$$


Liu [16] also defined the inverse function $\Phi^{-1}$ as the inverse uncertainty distribution of uncertain variable $\xi$.

Theorem 1. (Liu [16]) Let $\xi_{1}, \xi_{2}, \cdots, \xi_{n}$ be independent uncertain variables with regular uncertainty distributions $\Phi_{1}, \Phi_{2}, \cdots, \Phi_{n}$, respectively. If the function $f\left(x_{1}, x_{2}, \cdots, x_{n}\right)$ is strictly increasing with respect to $x_{1}, x_{2}, \cdots, x_{m}$ and strictly decreasing with respect to $x_{m+1}, x_{m+2}, \cdots, x_{n}$, then

$$
\xi=f\left(\xi_{1}, \xi_{2}, \cdots, \xi_{n}\right)
$$

is an uncertain variable with inverse uncertainty distribution

$$
\Phi^{-1}(\alpha)=f\left(\Phi_{1}^{-1}(\alpha), \cdots, \Phi_{m}^{-1}(\alpha), \Phi_{m+1}^{-1}(1-\alpha), \cdots, \Phi_{n}^{-1}(1-\alpha)\right) .
$$

Based on the definitions of uncertain variable and random variable, the concept of uncertain random variable is given as follows:

Definition 6. (Liu [25]) An uncertain random variable is a function $\xi$ from a chance space $(\Gamma, \mathcal{L}, \mathcal{M}) \times(\Omega, \mathcal{A}, \operatorname{Pr})$ to the set of real numbers such that $\{\xi \in B\}$ is an event in $\mathcal{L} \times \mathcal{A}$ for any Borel set $B$.

Example 1 A random variable is a special uncertain random variable since any real value is a special uncertain variable.

Example 2 The sum of a random variable and an uncertain variable is an uncertain random variable.

Definition 7. (Liu [25]) Let $(\Gamma, \mathcal{L}, \mathcal{M}) \times(\Omega, \mathcal{A}, \operatorname{Pr})$ be a chance space, and $\Theta \in \mathcal{L} \times \mathcal{A}$ be an event. Then, the chance measure of $\Theta$ is defined as

$$
\operatorname{Ch}\{\Theta\}=\int_{0}^{1} \operatorname{Pr}\{\omega \in \Omega \mid \mathcal{M}\{\gamma \in \Gamma \mid(\gamma, \omega) \in \Theta\} \geq x\} \mathrm{d} x .
$$

Liu [25] proved that the chance measure is self-dual.

Definition 8. (Liu [25]) Let $\xi$ be an uncertain random variable. Then, the chance distribution of $\xi$ is defined by

$$
\Phi(x)=\operatorname{Ch}\{\xi \leq x\}
$$

for any $x \in R$.

For some special case, some operational laws are presented as follows:

Theorem 2. (Liu [26]) Let $\eta_{1}, \eta_{2}, \cdots, \eta_{m}$ be independent random variables with probability distributions $\Psi_{1}, \Psi_{2}, \cdots, \Psi_{m}$, respectively, and $\tau_{1}, \tau_{2}, \cdots, \tau_{n}$ be uncertain variables (not necessarily independent). Then, the uncertain random variable $\xi=f\left(\eta_{1}, \eta_{2}, \cdots, \eta_{m}\right.$, $\left.\tau_{1}, \tau_{2}, \cdots, \tau_{n}\right)$ has a chance distribution

$$
\Phi(x)=\int_{R^{m}} F\left(x ; y_{1}, \cdots, y_{m}\right) \mathrm{d} \Psi_{1}\left(y_{1}\right) \cdots \mathrm{d} \Psi_{m}\left(y_{m}\right)
$$


where $F\left(x ; y_{1}, \cdots, y_{m}\right)$ is the uncertainty distribution of the uncertain variable $f\left(y_{1}, \cdots, y_{m}, \tau_{1}, \cdots, \tau_{n}\right)$ for any real numbers $y_{1}, \cdots, y_{m}$.

Theorem 3. (Liu [33]) Let $\eta_{1}, \eta_{2}, \cdots, \eta_{m}$ be independent random variables with probability distributions $\Psi_{1}, \Psi_{2}, \cdots, \Psi_{m}$, and $\tau_{1}, \tau_{2}, \cdots, \tau_{n}$ be independent uncertain variables with regular uncertainty distributions $\Upsilon_{1}, \Upsilon_{2}, \cdots, \Upsilon_{n}$, respectively. If $f\left(\eta_{1}, \cdots, \eta_{m}, \tau_{1}, \cdots, \tau_{n}\right)$ is strictly increasing with respect to $\tau_{1}, \cdots, \tau_{k}$ and strictly decreasing with respect to $\tau_{k+1}, \cdots, \tau_{n}$, then

$$
C h\left\{f\left(\eta_{1}, \cdots, \eta_{m}, \tau_{1}, \cdots, \tau_{n}\right) \leq 0\right\}=\int_{R^{m}} G\left(y_{1}, \cdots, y_{m}\right) \mathrm{d} \Psi_{1}\left(y_{1}\right) \cdots \mathrm{d} \Psi_{m}\left(y_{m}\right)
$$

where $G\left(y_{1}, \cdots, y_{m}\right)$ is the root $\alpha$ of the equation

$$
f\left(y_{1}, \cdots, y_{m}, \Upsilon_{1}^{-1}(\alpha), \cdots, \Upsilon_{k}^{-1}(\alpha), \Upsilon_{k+1}^{-1}(1-\alpha), \cdots, \Upsilon_{n}^{-1}(1-\alpha)\right)=0
$$

\section{Description of time-cost trade-off problem}

Whether a project is successful or not depends on the opinion of the responsible managers. Some of them may focus more on whether the project is on time or before. Some others may pay more attentions to the project budget. For some projects, e.g., China's manned space program, the highest priority is technical performance. Generally, the success of a project is measured by three dimensions, i.e., time, cost, and performance (quality or agreed-upon specifications). For many projects, since project managers only need to meet some quality standards, the dimension of quality is not so important for project success. In this case, project managers are required to make trade-off between project cost and project completion time with the project progress. Furthermore, project cost and project completion time are always in some relation. Sometimes, project managers may make decisions to finish projects sooner with project cost augment by accelerating project schedule, which is also named as project crashing in project management. In other cases, motivated by reducing project costs, managers may be conscripted to sacrifice with prolonging project completion times. To sum up, it is natural for project managers to find some schedules to complete projects with the balance of project costs and project completion times.

Generally, a project can be represented by an activity-on-arc (AoA) network $G=$ $(V, A)$, where $V=\{1,2, \cdots, n\}$ is the set of nodes representing the milestones and $A$ is the set of arcs representing the activities, shown as Figure 1. The normal duration of activity $(i, j)$ denoted as $\xi_{i j}$ represents the duration without the influence of decision made by the project manager. Correspondingly, the normal cost per time unit of activity $(i, j)$ is denoted by $c_{i j}$. The decision variable $x_{i j}$ indicates the duration change of activity $(i, j)$ controlled by the manager, which may be realized by determining work force change, instrument change, etc. The variable $x_{i j}$, supposed to be an integer for simplicity, is bounded by some interval $\left[l_{i j}, u_{i j}\right]$ owing to practical conditions, where $l_{i j}$ and $u_{i j}$ are also assumed to be integers. Similarly, the decision associated cost $d_{i j}$ is regarded as the additional cost of per unit change of $x_{i j}$. For simplicity, $c_{i j}$ and $d_{i j}$ are both assumed to be constants. Due to the changeable and indeterminate external environment, the normal durations $\xi_{i j}$ are always nondeterministic for all activities $(i, j) \in A$. For many projects, since the 'new' parts and ever performed parts coexist, the normal activity durations here are assumed to be uncertain random variables, concisely written as 


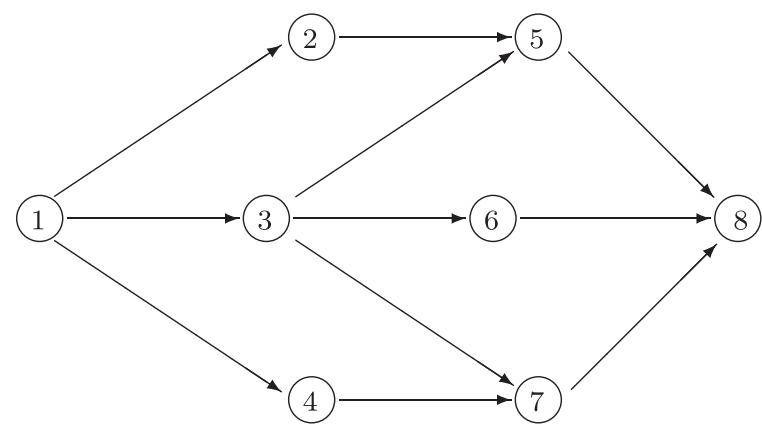

Figure 1 An illustration of a project.

$\xi=\left\{\xi_{i j}:(i, j) \in A\right\}$. For the trade-off between project completion time and project cost, the goal is to decide the optimal vector $\boldsymbol{x}=\left\{x_{i j}:(i, j) \in A\right\}$ to meet different scheduling requirements.

The starting time of activity $(i, j)$ is denoted by $T_{i j}(\boldsymbol{x}, \boldsymbol{\xi})$, and the starting time of activity $(1, j) \in A$ is defined as $T_{1 j}(\boldsymbol{x}, \boldsymbol{\xi})=0$, which means that the starting time of the total project is assumed to be 0 . To simplify the problem, it is assumed that each activity can be processed only if all the foregoing activities are finished and should be processed without interruption, lead times, and lag times. With the assumptions, the starting time of activity $(i, j), i=2,3, \cdots, n-1$ can be calculated by

$$
T_{i j}(\boldsymbol{x}, \boldsymbol{\xi})=\max _{(k, i) \in A}\left\{T_{k i}(\boldsymbol{x}, \boldsymbol{\xi})+\xi_{k i}+x_{k i}\right\}
$$

And the project completion time can be written by

$$
T(\boldsymbol{x}, \boldsymbol{\xi})=\max _{(k, n) \in A}\left\{T_{k n}(\boldsymbol{x}, \boldsymbol{\xi})+\xi_{k n}+x_{k n}\right\}
$$

The project cost, composed of the normal cost and the additional cost, can be calculated by

$$
C(\boldsymbol{x}, \boldsymbol{\xi})=\sum_{(i, j) \in A}\left(c_{i j} \xi_{i j}-d_{i j} x_{i j}\right)
$$

\section{Uncertain random time-cost trade-off model with dependent-chance programming}

Due to the complex project environment, achieving project goals faces many types of indeterminacy or risks, e.g., the weather variation and the government policy adjustment, leading to the fact that many project objectives cannot be obtained completely. Such a case may be more vital when project objectives are in conflict, as this is mostly the truth. Hence, being sure to maximize the opportunity of achieving project goals is as important as the trade-off between project goals in project management. In this section, dependent-chance programming (DCP), initiated by Liu [34], is applied in uncertain random TCTP. Generally, DCP is applied for risk-averse project managers to ensure the achievement of project goals for possibly extra expenses. Based 
on the DCP philosophy, the uncertain random chance maximization model is built as follows:

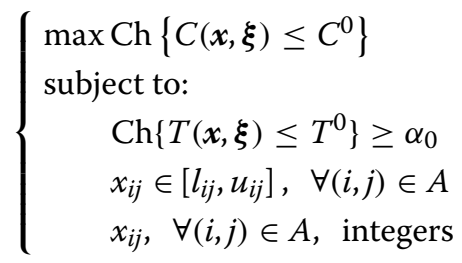

where $\alpha_{0}$ is the confidence level given in advance, $T^{0}$ is the due date of the project, $C^{0}$ is the budget, $l_{i j}$ and $u_{i j}$ are integers given in advance, and $T(\boldsymbol{x}, \boldsymbol{\xi})$ and $C(\boldsymbol{x}, \boldsymbol{\xi})$ are defined by Equations 1 and 2, respectively.

As we mentioned above, in most projects, 'new' parts and 'old' parts integrate into the whole project. That means the uncertain random environment for many projects can be divided into sole randomness and uncertainty. In this case, the uncertain random vector $\boldsymbol{\xi}$ can be represented with the form $\left(\eta_{1}, \eta_{2}, \cdots, \eta_{k}, \tau_{1}, \tau_{2}, \cdots, \tau_{l}\right)$, where $\eta_{1}, \eta_{2}, \cdots, \eta_{k}$ are independent random variables with probability distributions $\Psi_{1}, \Psi_{2}, \cdots, \Psi_{k}$, and $\tau_{1}, \tau_{2}, \cdots, \tau_{l}$ are independent uncertain variables with uncertainty distributions $\Upsilon_{1}$, $\Upsilon_{2}, \cdots, \Upsilon_{l}$, respectively. Since $C(\boldsymbol{x}, \boldsymbol{\xi})-C^{0}$ and $T(\boldsymbol{x}, \boldsymbol{\xi})-T^{0}$ are strictly increasing with respect to all the uncertain random variables, applying Theorem 3 , the above model can be transformed into the following crisp model:

$$
\left\{\begin{array}{l}
\max \int_{\mathcal{R}^{k}} G\left(\boldsymbol{x}, z_{1}, \cdots, z_{k}\right) \mathrm{d} \Psi_{1}\left(z_{1}\right) \cdots \mathrm{d} \Psi_{k}\left(z_{k}\right) \\
\text { subject to : } \\
\quad \int_{\mathcal{R}^{k}} H\left(\boldsymbol{x}, z_{1}, \cdots, z_{k}\right) \mathrm{d} \Psi_{1}\left(z_{1}\right) \cdots \mathrm{d} \Psi_{k}\left(z_{k}\right) \geq \alpha_{0} \\
\quad x_{i j} \in\left[l_{i j}, u_{i j}\right], \quad \forall(i, j) \in A \\
\quad x_{i j}, \quad \forall(i, j) \in A, \text { integers }
\end{array}\right.
$$

where $G\left(\boldsymbol{x}, z_{1}, \cdots, z_{k}\right)$ is the $\operatorname{root} \beta$ of

$$
C\left(x, z_{1}, \cdots, z_{k}, \Upsilon_{1}^{-1}(\beta), \cdots, \Upsilon_{l}^{-1}(\beta)\right)-C^{0}=0,
$$

and $H\left(\boldsymbol{x}, z_{1}, \cdots, z_{k}\right)$ is the root $\beta$ of

$$
T\left(\boldsymbol{x}, z_{1}, \cdots, z_{k}, \Upsilon_{1}^{-1}(\beta), \cdots, \Upsilon_{l}^{-1}(\beta)\right)-T^{0}=0 .
$$

\section{Algorithm description with numerical experiment}

Since Huang and Ding [31] demonstrated that the standard path algorithms were not able to approach the critical path for project scheduling problem with random activity durations, it is more difficult to solve uncertain random TCTP. Hence, uncertain random simulation and GA are integrated for solving the proposed chance maximization model in this section. An uncertain random simulation algorithm for chance measure (e.g., $\left.C h\left\{C(\boldsymbol{x}, \boldsymbol{\xi}) \leq C^{0}\right\}\right)$ can be obtained as follows:

\section{Uncertain random simulation for chance measure:}

Step 1. Set $e=0$.

Step 2. Randomly generate $\omega$ from the probability space according to the probability distribution. 
Step 3. $e \leftarrow e+\mathcal{M}\left\{C(\boldsymbol{x}, \boldsymbol{\xi}(\cdot, \omega)) \leq C^{0}\right\}$.

Step 4. Repeat the second and third steps $N$ times, where $N$ is a sufficiently large number.

Step 5. Return $e / N$ as the chance measure.

The above designed uncertain random simulation approach can be embedded in GA simply for solving the proposed model.

Now, consider a project illustrated in Figure 1. The activity durations, the normal costs, and the additional costs of the activities are presented in Table 1, respectively. Note that the uncertain random activity durations are partly linear uncertain variables with the form of $\mathcal{L}(a, b)$ and partly uniformly distributed random variables denoted by $\mathcal{U}(c, d)$, where $a<b$ and $c<d$ are crisp numbers. The chance maximization model is given as follows:

$$
\left\{\begin{array}{l}
\max \operatorname{Ch}\{C(\boldsymbol{x}, \boldsymbol{\xi}) \leq 24100\} \\
\text { subject to: } \\
\operatorname{Ch}\{T(\boldsymbol{x}, \boldsymbol{\xi}) \leq 35\} \geq 0.9 \\
x_{i j} \in[-4,3], \quad \forall(i, j) \in A \\
x_{i j}, \quad \forall(i, j) \in A, \text { integers. }
\end{array}\right.
$$

Uncertain random simulation and GA are employed to search for the optimal solution. For the above project, the parameters in GA are set as the population size 70, the mutation probability 0.8 , and the crossover probability 0.5 . After a run of 1,000 generations, the optimal solution and value are obtained: $\boldsymbol{x}^{*}=(1,-1,0,3,0,-3,1,3,-1,3,-1)$ and Ch $\{C(\boldsymbol{x}, \boldsymbol{\xi}) \leq 24100\}=0.938$, respectively.

\section{Conclusions}

In project management, one of the characteristics of the concept 'project' is that there is always something new in a project, i.e., a project always has some 'new' parts with little or no historical data. Meanwhile, most projects may contain some common or 'old' parts (with enough historical data) ever performed by many other finished projects. Hence, uncertainty and randomness should be considered simultaneously in project scheduling

Table 1 Activity durations and costs of project

\begin{tabular}{cccc}
\hline Activity & Normal duration & Normal cost & Additional cost \\
$(\boldsymbol{i}, \boldsymbol{j})$ & $\boldsymbol{\xi}_{\boldsymbol{i j}}$ & $\boldsymbol{c}_{\boldsymbol{i j}}$ & $\boldsymbol{d}_{\boldsymbol{i j}}$ \\
\hline$(1,2)$ & $\mathcal{L}(7,10)$ & 170 & 200 \\
$(1,3)$ & $\mathcal{U}(9,12)$ & 300 & 280 \\
$(1,4)$ & $\mathcal{L}(8,10)$ & 65 & 70 \\
$(2,5)$ & $\mathcal{U}(8,12)$ & 270 & 300 \\
$(3,5)$ & $\mathcal{L}(10,15)$ & 135 & 150 \\
$(3,6)$ & $\mathcal{L}(9,13)$ & 75 & 90 \\
$(3,7)$ & $\mathcal{U}(11,15)$ & 150 & 100 \\
$(4,7)$ & $\mathcal{L}(10,13)$ & 600 & 400 \\
$(5,8)$ & $\mathcal{U}(10,14)$ & 85 & 100 \\
$(6,8)$ & $\mathcal{L}(12,14)$ & 300 & 400 \\
$(7,8)$ & $\mathcal{U}(9,14)$ & 95 & 90 \\
\hline
\end{tabular}


problem. In this paper, an uncertain random time-cost trade-off model with dependentchance programming was built with a crisp equivalent model for the case that uncertain random parameters in the problem are partly random variables and partly uncertain variables. Furthermore, uncertain random simulation and GA were integrated for solving the proposed model.

The TCTP with uncertain random parameters can be regarded as the extension of the fuzzy TCTP, random TCTP, uncertain TCTP, and fuzzy random TCTP. Besides, since in this paper continuous time-cost trade-off relationship was assumed, in future research, discrete or mixed time-cost trade-off relationships can be introduced for modeling other project optimization problems.

\section{Acknowledgements}

The work was partly supported by the National Natural Science Foundation of China $(71371141,71001080)$.

Received: 22 September 2014 Accepted: 8 November 2014

Published online: 25 November 2014

\section{References}

1. Kelley, JE: Critical path planning and scheduling mathematical basis. Oper. Res. 9(3), 296-320 (1961)

2. Heberta, JE, Deckro, RF: Combining contemporary and traditional project management tools to resolve a project scheduling problem. Comput. Oper. Res. 38, 21-32 (2011)

3. Weglarz, J, Jozefowska, J, Mika, M, Waligora, G: Project scheduling with finite or infinite number of activity processing modes - A survey. Eur. J. Oper. Res. 208(3), 177-205 (2011)

4. Salmasnia, A, Mokhtari, H, Abadi, INK: A robust scheduling of projects with time, cost, and quality considerations. Int. J. Adv. Manuf. Tech. 60(5-8), 631-642 (2012)

5. Ghoddousi, P, Eshtehardian, E, Jooybanpour, S, Javanmardi, A: Multi-mode resource-constrained discrete time-cost-resource optimization in project scheduling using non-dominated sorting genetic algorithm. Automat. Constr. 30, 216-227 (2013)

6. Wollmer, RD: Critical path planning under uncertainty. Math. Program. Stud. 25, 164-171 (1985)

7. $\mathrm{Ke}, \mathrm{H}, \mathrm{Ma}, \mathrm{W}, \mathrm{Ni}, \mathrm{Y}$ : Optimization models and a GA-based algorithm for stochastic time-cost trade-off problem. Appl. Math. Comput. 215(1), 308-313 (2009)

8. Mokhtari, H, Aghaie, A, Rahimi, J, Mozdgir, A: Project time-cost trade-off scheduling: a hybrid optimization approach. Int. J. Adv. Manuf. Tech. 50, 811-822 (2010)

9. $\mathrm{Ke}, \mathrm{H}, \mathrm{Ma}, \mathrm{W}, \mathrm{Chen}, \mathrm{X}$ : Modeling stochastic project time-cost trade-offs with time-dependent activity durations. Appl. Math. Comput. 218(18), 9462-9469 (2012)

10. Leu, SS, Chen, AT, Yang, CH: A GA-based fuzzy optimal model for construction time-cost trade-off. Int. J. Proj. Manage. 19, 47-58 (2001)

11. Ghazanfari, M, Yousefli, A, Ameli, MSJ, Bozorgi-Amiri, A: A new approach to solve time-cost trade-off problem with fuzzy decision variables. Int. J. Adv. Manuf. Tech. 42, 408-414 (2009)

12. Ke, H, Ma, W, Gao, X, Xu, W: New fuzzy models for time-cost trade-off problem. Fuzzy Optim. Decis. Ma. 9(2), 219-231 (2010)

13. Chen, S, Tsai, M: Time-cost trade-off analysis of project networks in fuzzy environments. Eur. J. Oper. Res. 212(2), 386-397 (2011)

14. Liu, B: Why is there a need for uncertainty theory. J. Uncertain Syst. 6, 3-10 (2012)

15. Liu, B: Uncertainty Theory. 2nd ed. Springer-Verlag, Berlin (2007)

16. Liu, B: Uncertainty Theory: A Branch of Mathematics for Modeling Human Uncertainty. Springer-Verlag, Berlin (2010)

17. Bhattacharyya, R, Chatterjee, A, Kar, S: Uncertainty theory based multiple objective mean-entropy-skewness stock portfolio selection model with transaction costs. J. Uncertain. Anal. Appl. 1, Article 16 (2013)

18. Ding, S: Uncertain random newsboy problem. J. Intell. Fuzzy Syst. 26, 483-490 (2014)

19. Gao, Y: Uncertain models for single facility location problems on networks. Appl. Math. Model. 36, 2592-2599 (2012)

20. Jana, DK, Maity, K, Roy, TK: A three-layer supply chain integrated production-inventory model under permissible delay in payments in uncertain environments. J. Uncertain. Anal. Appl. 1, Article 6 (2013)

21. Lu, Y, Huang, Z, Gao, J: Under-investment problem with adverse selection in uncertain environment. J. Uncertain Syst. 8, 83-89 (2014)

22. Yang, $X, G a o, J:$ Uncertain differential games with application to capitalism. J. Uncertain. Anal. Appl. 1, Article 17 (2013)

23. Yang, X, Gao, J: Uncertain core for coalitional game with uncertain payoffs. J. Uncertain Syst. 8, 13-21 (2014)

24. Ke, H: A genetic algorithm-based optimizing approach for project time-cost trade-off with uncertain measure. J. Uncertain. Anal. Appl. 2, Article 8 (2014)

25. Liu, YH: Uncertain random variables: A mixture of uncertainty and randomness. Soft. Comput. 17, 625-634 (2013)

26. Liu, YH: Uncertain random programming with applications. Fuzzy Optim. Decis. Ma. 12, 153-169 (2013)

27. Liu, B: Uncertain Random Graph and Uncertain Random Network. J. Uncertain Syst. 8, 3-12 (2014)

28. Liu, YH, Ralescu, DA: Risk index in uncertain random risk analysis. Int. J. Uncertain. Fuzz. 22, 491-504 (2014)

29. Zhou, J, Yang, F, Wang, K: Multi-objective optimization in uncertain random environments. Fuzzy Optim. Decis. Ma. 13, 397-413 (2014) 
30. $\mathrm{Ke}, \mathrm{H}, \mathrm{Su}, \mathrm{T}, \mathrm{Ni}, \mathrm{Y}$ : Uncertain random multilevel programming with application to production control problem (2014). doi:10.1007/s00500-014-1361-2

31. Huang, W, Ding, L: Project-scheduling problem with random time-dependent activity duration times. IEEE T. Eng Manage. 58, 377-387 (2011)

32. Liu, B: Some research problems in uncertainty theory. J. Uncertain Syst. 3, 3-10 (2009)

33. Liu, YH, Ralescu, DA: Risk index in uncertain random risk analysis. Int. J. Uncertain. Fuzz. 22, 491-504 (2014)

34. Liu, B: Dependent-chance programming: a class of stochastic programming. Comput. Math. Appl. 34, 89-104 (1997)

doi:10.1186/s40467-014-0023-3

Cite this article as: Ke: Uncertain random time-cost trade-off problem. Journal of Uncertainty Analysis and Applications $20142: 23$.

Submit your manuscript to a SpringerOpen ${ }^{\odot}$ journal and benefit from:

- Convenient online submission

- Rigorous peer review

- Immediate publication on acceptance

- Open access: articles freely available online

- High visibility within the field

- Retaining the copyright to your article

Submit your next manuscript at $\boldsymbol{\triangleright}$ springeropen.com 